Pacific

Journal of

Mathematics

UPPER BOUNDS FOR THE NUMBER OF LIMIT CYCLES THROUGH LINEAR DIFFERENTIAL EQUATIONS

ARmengol Gasull and Hector Giacomini 


\title{
UPPER BOUNDS FOR THE NUMBER OF LIMIT CYCLES THROUGH LINEAR DIFFERENTIAL EQUATIONS
}

\author{
Armengol Gasull And Hector Giacomini
}

\begin{abstract}
Consider the differential equation $\dot{x}=y, \dot{y}=h_{0}(x)+h_{1}(x) y+h_{2}(x) y^{2}+y^{3}$ in the plane. We prove that if a certain solution of an associated linear ordinary differential equation does not change sign, there is an upper bound for the number of limit cycles of the system. The main ingredient of the proof is the Bendixson-Dulac criterion for $\ell$-connected sets. Some concrete examples are developed.
\end{abstract}

\section{Main results}

Although second order ordinary differential equations of the form $\ddot{x}=f(x, \dot{x})$ are some of the easiest autonomous planar differential equations, most problems concerning the study of the number of periodic solutions remain open. For instance, even if we consider the Kukles system $\dot{x}=y, \dot{y}=f_{3}(x, y)$, where $f_{3}$ is a polynomial of degree at most 3 , the maximum number of limit cycles that it can have is still unknown.

This paper deals with the problem of finding methods to establish upper bounds for the number of limit cycles of planar differential equations of the form

$$
\dot{x}=y, \quad \dot{y}=h_{0}(x)+h_{1}(x) y+h_{2}(x) y^{2}+y^{3},
$$

where the functions $h_{i}$ are smooth enough.

The proof of our main result is based on the use of the generalized BendixsonDulac criterion for $\ell$-connected sets. Recall that an open subset $U$ of $\mathbb{R}^{2}$ is said to be $\ell$-connected if its fundamental group $\pi_{1}(U)$ is the free group in $\ell$ generators. This method has already been used with similar goals by several authors; see for instance [Cherkas 1997; Lloyd 1979; Yamato 1979; Cherkas and Grin' 1997; 1998; Gasull and Giacomini 2002]. The novelty of our approach is that we are able to reduce the computation of an upper bound for the number of limit cycles of the

MSC2000: 34C07, 34C05, 34A30, 37C27.

Keywords: ordinary differential equation, limit cycle, Bendixson-Dulac criterion, linear ordinary differential equation.

The first author is partially supported by the DGES grant number BFM2002-04236 and CONACIT grant number 2001SGR-00173. 
differential system (1-1) to the study of the positiveness of a certain function, which has the important property of satisfying a linear ordinary differential equation.

More precisely, our main result is this:

Theorem A. Let $X$ be the vector field associated with the differential equation

$$
\dot{x}=y, \quad \dot{y}=h_{0}(x)+h_{1}(x) y+h_{2}(x) y^{2}+y^{3},
$$

and fix a positive integer number $n$. Then there exists a constructive procedure, detailed in Lemmas 2.2 and 2.4, to associate with $X$ two functions $f_{n}(x, y)$ and $M_{n}(x)$, such that

(i) $\left.\operatorname{div}\left(\left|f_{n}(x, y)\right|^{-3 / n} X(x, y)\right)=-\frac{3}{n} \operatorname{sgn}\left(f_{n}(x, y)\right) \mid f_{n}(x, y)\right)\left.\right|^{-1-3 / n} M_{n}(x)$, and

(ii) the function $y=M_{n}(x)$ is defined for all $x \in \mathbb{R}$ and is a solution of a linear ordinary differential equation of the form

$$
s_{n, n+1}(x) y^{(n+1)}(x)+s_{n, n}(x) y^{(n)}(x)+\cdots+s_{n, 1}(x) y^{\prime}(x)+s_{n, 0}(x) y(x)=0 .
$$

Assume furthermore that $M_{n}(x)$ does not change sign and vanishes only at finitely many points. Then:

(iii) The limit cycles of (1-2) do not cut the curves $\left\{f_{n}(x, y)=0\right\}$.

(iv) The number of limit cycles of (1-2) contained in an $\ell$-connected component $U$ of $\mathbb{R}^{2} \backslash\left\{f_{n}(x, y)=0\right\}$ is at most $\ell$. All these limit cycles are hyperbolic and their stability is given by the sign of $M_{n}(x)$ and the sign of $f_{n}(x, y)$ in the region occupied by the limit cycle.

From the proof of the theorem it is easy to observe that, with small modifications, it can also be applied to systems for which the second equation is $\dot{y}=h_{0}(x)+$ $h_{1}(x) y+h_{2}(x) y^{2}+h_{3}(x) y^{3}$.

In Section 3 we study a simple example, the van der Pol equation, to show how the method works.

In Section 4 we study the number of limit cycles of the system

$$
\dot{x}=y, \quad \dot{y}=-x^{3}+d x y^{2}+y^{3} .
$$

For this system the expected upper bound is of one limit cycle, but as far as we know this is still an open question. The authors have studied this problem by using several existing methods in the literature but no progress has been possible. For this reason we have selected this problem to test the effectiveness of the new method proposed. A motivation for its study is also given at the beginning of Section 4 . The results obtained are detailed in Section 5.

In these two examples we see that we can reduce the study of the number of limit cycles of a planar polynomial system to the study of a linear ordinary differential equation. Although the study of this last equation is not easy and requires special 
tricks for each concrete application, it provides a new way for trying to control the number of limit cycles for special classes of planar polynomial systems. Also, for the main example developed in Section 4, we can see that the final step goes to a one-variable nonlinear equation. To end this introduction we would like to stress this last scheme:

$$
\begin{gathered}
\text { Planar ordinary } \\
\text { nonlinear differential equation }
\end{gathered} \longrightarrow \begin{gathered}
\text { Linear ordinary } \\
\text { differential equation }
\end{gathered} \longrightarrow \begin{gathered}
\text { Nonlinear } \\
\text { equation }
\end{gathered}
$$

\section{Preliminary results and proof of Theorem A}

First we recall the generalized Bendixson-Dulac criterion. For various proofs, see [Lloyd 1979; Yamato 1979; Gasull and Giacomini 2002].

Proposition 2.1 (Generalized Bendixson-Dulac Criterion). Consider a $\mathscr{C}^{1}$ differential system

$$
\dot{x}=P(x, y), \quad \dot{y}=Q(x, y),
$$

and set $X=(P, Q)$. Let $U$ be an open $\ell$-connected subset of $\mathbb{R}^{2}$ with a smooth boundary. Assume that

$$
\operatorname{div}(X)=\frac{\partial P}{\partial x}+\frac{\partial Q}{\partial y}
$$

does not change sign on $U$ and vanishes only on a null measure Lebesgue set. Then the system can have at most $\ell$ periodic orbits contained in $U$. Each such orbit is hyperbolic and its stability is given by the sign of $\operatorname{div}(X)$.

We now turn to preliminary computations needed to prove Theorem A.

Lemma 2.2. Consider the system (1-2),

$$
\begin{aligned}
& \dot{x}=y=: P(x, y), \\
& \dot{y}=h_{0}(x)+h_{1}(x) y+h_{2}(x) y^{2}+y^{3}=: Q(x, y),
\end{aligned}
$$

and fix a positive integer number $n$. There is a constructive procedure to find $n+1$ functions $r_{n, i}(x), i=0, \ldots, n$, satisfying the following condition:

Let $y(x)=g_{n}(x)$ be any solution of the order- $(n+1)$ linear ordinary differential equation

$$
y^{(n+1)}(x)+r_{n, n}(x) y^{(n)}(x)+\cdots+r_{n, 1}(x) y^{\prime}(x)+r_{n, 0}(x) y(x)=0,
$$

and let $g_{n, i}(x)$, where $i=0, \ldots, n-1$, be defined in terms of $h_{0}(x), h_{1}(x)$, $h_{2}(x), g_{n}(x)$, their derivatives, and $g_{n, n}(x):=g_{n}(x)$. Then, setting

$$
f_{n}(x, y):=g_{n, 0}(x)+g_{n, 1}(x) y+g_{n, 2}(x) y^{2}+\cdots+g_{n, n}(x) y^{n},
$$

the expression

$$
M_{n}:=\left\langle\nabla f_{n},(P, Q)\right\rangle-\frac{n}{3} f_{n} \operatorname{div}(P, Q)
$$


is a function of only the $x$-variable.

Proof. Instead of dealing with a general $n$ and for the sake of clarity, we present the details of the proof just for the case $n=2$. The general case can be handled in the same way. Also, for the sake of brevity and during this proof, when a function of $x$ appears that we do not want to specify, we will write $*$.

Take $f_{2}(x, y)=g_{2,0}(x)+g_{2,1}(x) y+g_{2,2}(x) y^{2}:=g_{0}(x)+g_{1}(x) y+g_{2}(x) y^{2}$. Then,

$$
\begin{aligned}
M_{2}(x, y)=\langle\nabla & \left.f_{2},(P, Q)\right\rangle-\frac{2}{3} \operatorname{div}(P, Q) f_{2} \\
=\left(g_{2}^{\prime}(x)+\frac{2}{3} g_{2}(x) h_{2}(x)-g_{1}(x)\right) y^{3} & \quad+\left(g_{1}^{\prime}(x)+\frac{4}{3} g_{2}(x) h_{1}(x)-\frac{1}{3} g_{1}(x) h_{2}(x)-2 g_{0}(x)\right) y^{2} \\
& +\left(g_{0}^{\prime}(x)+\frac{1}{3} g_{1}(x) h_{1}(x)-\frac{4}{3} h_{2}(x) g_{0}(x)+2 g_{2}(x) h_{0}(x)\right) y \\
& +\left(g_{1}(x) h_{0}(x)-\frac{2}{3} h_{1}(x) g_{0}(x)\right) .
\end{aligned}
$$

By choosing

$$
\begin{aligned}
& g_{0}(x)=\frac{1}{2}\left(g_{1}^{\prime}(x)+\frac{4}{3} g_{2}(x) h_{1}(x)-\frac{1}{3} g_{1}(x) h_{2}(x)\right), \\
& g_{1}(x)=g_{2}^{\prime}(x)+\frac{2}{3} g_{2}(x) h_{2}(x),
\end{aligned}
$$

we ensure that the coefficients of $y^{2}$ and $y^{3}$ in $M_{2}$ vanish. Observe that $g_{1}(x)=$ $g_{2}^{\prime}(x)+* g_{2}(x)$ and that $g_{0}(x)=g_{2}^{\prime \prime}(x) / 2+* g_{2}^{\prime}(x)+* g_{2}(x)$. If we substitute these equalities into the coefficient of $y$ in the expression for $M_{2}$, we obtain $g_{2}^{\prime \prime \prime}(x) / 2+$ $* g_{2}^{\prime \prime}(x)+* g_{2}^{\prime}(x)+* g_{2}(x)$. By imposing that this last expression be identically zero, we get the linear ordinary differential equation (2-1) given in the statement of the lemma. Hence for these values of the functions $g_{i}$, where $i=0,1,2$, the expression of $M_{2}$ is the function of one variable

$$
M_{2}(x)=g_{1}(x) h_{0}(x)-\frac{2}{3} h_{1}(x) g_{0}(x),
$$

as we wanted to prove.

Remark 2.3. (i) From the proof of Lemma 2.2 it is easy to observe that if all the functions $h_{i}$ appearing in system (1-2) are polynomials, then all the functions $r_{n, i}$ are polynomials as well.

(ii) If in system (1-2) instead of considering $\dot{y}=h_{0}(x)+h_{1}(x) y+h_{2}(x) y^{2}+y^{3}$, we take $\dot{y}=h_{0}(x)+h_{1}(x) y+h_{2}(x) y^{2}+h_{3}(x) y^{3}$, then a similar result can be proved. The main difference is that the function $h_{3}$ and its powers appear in the denominators of the expressions of $r_{n, i}$. Hence, all the computations make sense on only the strips where $h_{3}(x)$ does not vanish. 
Lemma 2.4. Let $M_{n}(x)$ be the one-variable function described in Lemma 2.2. Then there exists an order- $(n+1)$ linear ordinary differential equation

$$
\text { (2-4) } s_{n, n+1}(x) y^{(n+1)}(x)+s_{n, n}(x) y^{(n)}(x)+\cdots+s_{n, 1}(x) y^{\prime}(x)+s_{n, 0}(x) y(x)=0,
$$

such that $y=M_{n}(x)$ is one of its solutions. Here the functions $s_{n, i}$, for $i=0, \ldots$, $n+1$, can be explicitly obtained from all the functions appearing in Lemma 2.2.

Proof. As for Lemma 2.2, we detail the proof just for the case $n=2$. We continue denoting a generic smooth function of the variable $x$ by $*$. From (2-3) and (2-2), we have $M:=M_{2}=* g_{2}^{\prime \prime}+* g_{2}^{\prime}+* g_{2}$ and from the proof of Lemma 2.2 we obtain $g_{2}^{\prime \prime \prime}(x)+* g_{2}^{\prime \prime}(x)+* g_{2}^{\prime}(x)+* g_{2}(x)=0$. Hence, if we differentiate the first equality three times and the second one twice, we get the linear system

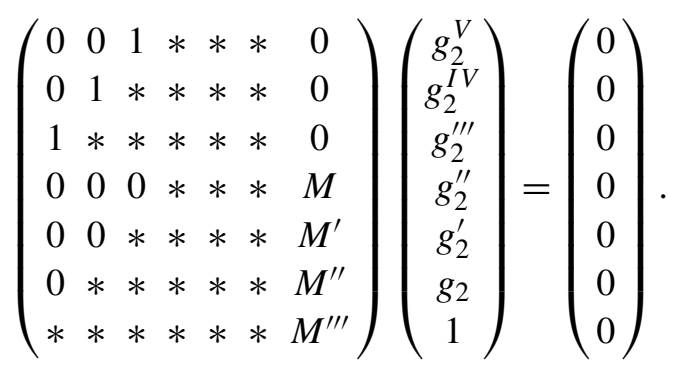

Since for all $x$, this system has the nonzero solution given by the function $g_{2}(x)$ and its derivatives, the determinant of the matrix is identically zero. By developing it from its last column, we get the linear ordinary differential equation satisfied by $M$ given in the statement of the lemma.

Remark 2.5. A main difference between the linear differential equations of order $n+1$ satisfied by $g_{n}$ and $M_{n}$ and those given in (2-1) and (2-4), respectively, is that the coefficient of the highest order derivative is identically 1 in the first case, and a function of $x$ in the second case. Hence, one could think that $M_{n}$ is not defined for all $x$, but this is not the case because it is also given by (2-3). In other words, $M_{n}$ is a solution of the linear equation (2-4) defined for all real $x$.

Proof of Theorem A. (i) From Lemma 2.2 we can construct a function $f_{n}(x, y)$ such that

$$
M_{n}:=\left\langle\nabla f_{n}, X\right\rangle-\frac{n}{3} \operatorname{div}(P, Q) f_{n}
$$

depends just on $x$, as we wanted to prove.

(ii) By Lemma 2.4, the function $y=M_{n}(x)$ satisfies the linear ordinary differential equation (2-4). Furthermore, by Remark 2.5 we know that it is defined for all $x \in \mathbb{R}$.

Assume from now on that $M_{n}(x)$ does not change sign and vanishes only on a finite set of points. 
(iii) Since

$$
\left.M_{n}\right|_{f_{n}=0}=\left.\left\langle\nabla f_{n}, X\right\rangle\right|_{f_{n}=0},
$$

from the control on the sign of $M_{n}$, the periodic orbits of (1-2) never cut the curves $\left\{f_{n}=0\right\}$, because the flow associated with $X$ crosses each one of them either inwards or outwards.

(iv) Instead of considering the vector field $X$, we take the new one $\left|f_{n}\right|^{-3 / n} X$. From the previous paragraph, we know that none of the limit cycles of $X$ intersect $\left\{f_{n}=0\right\}$. Hence each limit cycle is contained in a connected component $U$ of $\mathbb{R}^{2} \backslash\left\{f_{n}=0\right\}$. Note that

$$
\begin{aligned}
\operatorname{div}\left(\left|f_{n}\right|^{-3 / n} X\right) & =\left\langle\nabla\left(\left|f_{n}\right|^{-3 / n}\right), X\right\rangle+\left|f_{n}\right|^{-3 / n} \operatorname{div}(X) \\
& =-\frac{3}{n} \operatorname{sgn}\left(f_{n}\right)\left|f_{n}\right|^{-1-3 / n}\left\langle\nabla f_{n}, X\right\rangle+\left|f_{n}\right|^{-3 / n} \operatorname{div}(X) \\
& =-\frac{3}{n} \operatorname{sgn}\left(f_{n}\right)\left|f_{n}\right|^{-1-3 / n}\left(\left\langle\nabla f_{n}, X\right\rangle-\frac{1}{3} n f_{n} \operatorname{div}(X)\right) \\
& =-\frac{3}{n} \operatorname{sgn}\left(f_{n}\right)\left|f_{n}\right|^{-1-3 / n} M_{n} .
\end{aligned}
$$

Therefore, $\operatorname{div}\left(\left|f_{n}\right|^{-3 / n} X\right)$ does not change sign on $U$. By using the generalized Bendixson-Dulac criterion (Proposition 2.1), the theorem follows.

\section{A first example: the van der Pol equation}

The uniqueness of the limit cycle of the van der Pol equation can be proved by several different methods. We have chosen this simple example to illustrate our approach. Recall that the van der Pol equation is

$$
\dot{x}=y-\varepsilon\left(\frac{1}{3} x^{3}-x\right), \quad \dot{y}=-x .
$$

It can be transformed into the form (1-2) by interchanging $x$ and $y$ and then changing $y$ to $-y$. This gives

$$
\dot{x}=y, \quad \dot{y}=-x-\varepsilon\left(\frac{1}{3} y^{3}-y\right) .
$$

To prove the uniqueness of the limit cycle of this system by our method, we will apply Theorem A with $n=2$. Notice that since $f_{2}(x, y)=g_{0}(x)+g_{1}(x) y+$ $g_{2}(x) y^{2}$, for this value of $n$ all the connected components of $\mathbb{R}^{2} \backslash\left\{f_{2}=0\right\}$ are either simply connected or 1-connected. Furthermore, there is at most one 1-connected component that surrounds the origin.

With the notation introduced in Theorem A, we have

$$
\begin{aligned}
g_{0}(x) & =\frac{3}{2}\left(3 g_{2}^{\prime \prime}(x)-\frac{4}{3} g_{2}(x)\right), \quad g_{1}(x)=-3 g_{2}^{\prime}(x), \\
M_{2}(x) & =\frac{1}{3}\left(4 g_{2}(x)+9 x g_{2}^{\prime}(x)-9 g_{2}^{\prime \prime}(x)\right),
\end{aligned}
$$


where we have taken $\varepsilon=1$ to simplify the calculations. The function $g_{2}(x)$ is any solution of the third order linear differential equation

$$
9 y^{\prime \prime \prime}(x)-6 y^{\prime}(x)-4 x y(x)=0 .
$$

We have to choose a suitable solution $g_{2}$ such that its associated $M_{2}$ does not change sign. From the classical theory of linear differential equations, (see for instance [Ince 1927; Wasow 1965]), the solutions of this equation are analytic and entire. We can write them as

$$
g_{2}(x)=\sum_{n=0}^{\infty} a_{n} x^{n},
$$

with $a_{3}=\frac{1}{9} a_{1}$ and

$$
a_{n}=\frac{4 a_{n-4}+6(n-2) a_{n-2}}{9 n(n-1)(n-2)}, \text { for } n \geq 4 .
$$

For facilitating the control of the sign of $M_{2}(x)$ we take the even solution of (3-3), defined by the conditions

$$
y(0)=1, \quad y^{\prime}(0)=0, \quad y^{\prime \prime}(0)=0 .
$$

With these initial conditions, it is clear from (3-4) that all the nonzero coefficients $a_{n}$ are positive. Hence, $g_{2}$ as well as all of its derivatives, are positive for positive $x$. Furthermore, from (3-2) we see that $M_{2}$ is an even function with $M_{2}(0)=4 / 3$. Hence, it suffices to study the sign of $M_{2}(x)$ for $x>0$. Let us prove that the $m$ th derivative of $M_{2}(x)$ is positive for $x>0$ and any $m \geq 3$. By taking the third derivative of $M_{2}(x)$ from (3-2), and by using (3-3), we obtain

$$
M_{2}^{\prime \prime \prime}(x)=\frac{2}{3}\left(\frac{68}{9} x g_{2}(x)+\left(2 x^{2}+\frac{13}{3}\right) g_{2}^{\prime}(x)+x g_{2}^{\prime \prime}(x)\right) .
$$

This equality and the properties of $g_{2}(x)$ and its derivatives imply that $M_{2}^{\prime \prime \prime}(x)>0$ for all positive $x$. Furthermore, by taking more derivatives of this expression, and using the equality $g_{2}^{\prime \prime \prime}(x)=\frac{1}{9}\left(6 g_{2}^{\prime}(x)+4 x g_{2}(x)\right)$ at each step, we obtain only positive coefficients during all of the computations. Hence, our assertion follows about the derivatives of $M_{2}$. By using Taylor's Formula and some straightforward computations, we get

$$
M_{2}(x)=\frac{4}{3}-\frac{2}{3} x^{2}+\frac{17}{81} x^{4}+\frac{1}{5 !} M_{2}^{(5)}\left(s_{x}\right) x^{5},
$$

for some $s_{x}$ between 0 and $x$. Hence, for $x>0$,

$$
M_{2}(x) \geq \frac{4}{3}-\frac{2}{3} x^{2}+\frac{17}{81} x^{4}>0,
$$


as we wanted to prove. As a consequence of Theorem A, we conclude that the system (3-1) has at most one limit cycle. Moreover, when this limit cycle exists, it is hyperbolic and stable.

The van der Pol equation has a unique critical point located at the origin and it is repelling for $\varepsilon>0$. The point at infinity is also a repeller for $\varepsilon>0$. Therefore, the system has at least one limit cycle. Combining both statements we have proved the existence, uniqueness and hyperbolicity of the limit cycle.

We remark that in order to find an upper bound for the number of limit cycles by using our approach, it has not been necessary to explicitly solve the linear differential equation satisfied by the function $g_{2}(x)$. Only general properties of this function, which can be easily obtained from the linear equation, have been employed. In the next section we analyze a more difficult case.

\section{A second example}

We start this section with some motivation for the system of ordinary differential equations that we will study. In [Cima et al. 1997] it is proved that there are systems of the form

$$
\dot{x}=P_{2 n+1}(x, y), \quad \dot{y}=Q_{2 m+1}(x, y),
$$

with $P_{2 n+1}$ and $Q_{2 m+1}$ homogeneous polynomials of degrees $2 n+1$ and $2 m+$ 1 respectively $(n \neq m)$, possessing at least $n+m+1$ limit cycles surrounding the origin. These examples are constructed by studying the perturbations of the Hamiltonian system $\dot{x}=y^{2 n+1}, \dot{y}=-x^{2 m+1}$. Inside this family, the simplest case, $n=0$ and $m=1$, gives a system of the form

$$
\dot{x}=a x+b y, \quad \dot{y}=c x^{3}+d x^{2} y+e x y^{2}+f y^{3}
$$

with at least two limit cycles. We would like to investigate whether there can be more than two. This seems to be a hard problem, and so we start by considering the simplest case:

$$
\dot{x}=y, \quad \dot{y}=-x^{3}+d x^{2} y+y^{3},
$$

for which it is not difficult to prove that there is at least one limit cycle [Cima et al. 1997]. This section is devoted to trying to prove that in fact one is the maximum number of limit cycles that the system can have. Before starting our study, we want to comment that we have not been able to prove the uniqueness of the limit cycles of system (4-1) by using standard results in the literature, such as those in [Ye et al. 1986; Zhang et al. 1992]. Our results are summarized in Section 5. As a starting point we prove a previous result that reduces the study to the case $d<0$.

Lemma 4.1. (i) The origin is the only critical point of the system (4-1).

(ii) If $d \geq 0$, the system has no limit cycles. 
(iii) For $d<0$ is close enough to zero, the system has at least one limit cycle.

(iv) If for some $\bar{d}<0$ the system has no limit cycles, the same holds for any $d \leq \bar{d}$. Proof. Part (i) is trivial.

(ii) The divergence of $X=(P, Q)$, the vector field associated with (4-1), equals $d x^{2}+3 y^{2}$. For $d \geq 0$ this is always positive or zero, so using the divergence criterion we deduce that system (4-1) has no limit cycles.

(iii) Notice that (4-1) is a semicomplete family of rotated vector fields with respect to the parameter $d$, or SCFRVF for short (see [Duff 1953; Perko 1975]). This follows from the next computations, where we denote the vector field associated with (4-1) by $X_{d}(x, y)=\left(P_{d}(x, y), Q_{d}(x, y)\right)$,

$$
\begin{aligned}
\frac{\partial}{\partial d} \arctan \frac{Q_{d}(x, y)}{P_{d}(x, y)} & =\frac{P_{d}(x, y) \partial Q_{d}(x, y) / \partial d-Q_{d}(x, y) \partial P_{d}(x, y) / \partial d}{P_{d}^{2}(x, y)+Q_{d}^{2}(x, y)} \\
& =\frac{x^{2} y^{2}}{P_{d}^{2}(x, y)+Q_{d}^{2}(x, y)} \geq 0 .
\end{aligned}
$$

In [Cima et al. 1997] it is proved that the origin is a repeller when $d \geq 0$, and an attractor when $d<0$. Combining this with the fact that our system is an SCFRVF, we see that a repelling limit cycle bifurcates from the origin when $d$ is negative and small. Hence, item (iii) follows.

To prove item (iv) we need to recall more properties of an SCFRVF. The first is the nonintersection property, which asserts that limit cycles corresponding to different values of $d$ are disjoint.

The second is the planar termination principle [Perko 1990a; 1990b], which asserts the following for polynomial families of an SCFRVF: If $d$ varies and we consider the continuous evolution of some limit cycle born at a critical point $p$ (allowing for the possibility that the limit cycle goes to a multiple limit cycle, in which case we continue with the other limit cycle that has collided with it), then the union of this one-parameter family of limit cycles is a 1-connected open set $K$ whose boundaries are $p$ and a cycle of separatrices of $X_{d}$. The corners of this cycle of separatrices are finite or infinite critical points of $X_{d}$. In our case, because the only finite critical point of $X_{d}$ is the origin, $K$ is unbounded.

If for some value of $d=\bar{d}<0$ the system has no limit cycles, this means that the limit cycles starting at the origin for $d=0$ have disappeared for some $d^{*}$, where $\bar{d}<d^{*}<0$, covering the set $K$. Since $K$ covers from a neighborhood of the origin until infinity, then by the nonintersection property, the limit cycle cannot exist for $d \leq \bar{d}$ either, as we wanted to prove.

Nonexistence of limit cycles. We will now find a value $d=\bar{d}$, as sharp as possible, that determines parameter values for which there are no limit cycles. In a later section (page 289) we will study when there is a unique limit cycle. 
From Lemma 4.1, to complete the study of the nonexistence of limit cycles for (4-1), the case $d<0$ remains:

$$
\dot{x}=y, \quad \dot{y}=-x^{3}-a^{2} x^{2} y+y^{3} .
$$

To prove the nonexistence of limit cycles, it suffices to apply Theorem A with $n=1$, because for this value of $n$ all of the connected components of $\mathbb{R}^{2} \backslash\left\{f_{1}=0\right\}$ are simply connected. With the notation introduced in this theorem we have

$$
M_{1}(x)=-x^{2}\left(x g_{1}(x)-\frac{1}{3} a^{2} g_{1}^{\prime}(x)\right),
$$

where $g_{1}(x)$ is any solution of the linear ordinary differential equation

$$
y^{\prime \prime}(x)-\frac{2}{3} a^{2} x^{2} y(x)=0 .
$$

To conclude the nonexistence of limit cycles, it suffices to give a concrete solution $g_{1}$ of (4-3) such that the corresponding $M_{1}$ does not change sign. In fact it suffices to ensure that

$$
h_{a}(x):=x g_{1}(x)-\frac{1}{3} a^{2} g_{1}^{\prime}(x)
$$

does not change sign. Furthermore, from Theorem A we also know that $M_{1}$ satisfies a second order linear differential equation. Hence, the same happens with $h_{a}$. The expression $h_{a}(x)$ satisfies the equation

$$
\begin{aligned}
\left(\left(6 a^{6}-81\right) x^{2}-27 a^{2}\right) y^{\prime \prime}(x)-6\left(2 a^{6}-27\right) x y^{\prime}(x) & \\
& -\left(\left(4 a^{8}-54 a^{2}\right) x^{4}-18 a^{4} x^{2}+162\right) y(x)=0 .
\end{aligned}
$$

Fortunately, we can solve (4-3) in terms of the modified Bessel functions. More concretely, for all $x \in \mathbb{R}^{+}$, define

$$
I_{\nu}(x):=\sum_{k=0}^{\infty} \frac{(x / 2)^{v+2 k}}{\Gamma(v+k+1) \Gamma(k+1)} .
$$

This function is a solution of the Bessel equation

$$
x^{2} y^{\prime \prime}(x)+x y^{\prime}(x)-\left(x^{2}+v^{2}\right) y(x)=0 .
$$

Hence, it is easy to check that

$$
g_{1}(x)=\sqrt{x} I_{1 / 4}\left(\frac{a x^{2}}{\sqrt{6}}\right)
$$

is a solution of (4-3) for $x>0$, and it can be extended to an odd solution of (4-3) for all $x \in \mathbb{R}$. 
From now on we will fix this choice for $g_{1}$. In Lemma 4.2 we collect some known properties of the modified Bessel functions that are useful for our study of $h_{a}$. Before stating them we need to introduce some standard notations.

Following Poincaré's definition, given a function $f: \mathbb{R} \rightarrow \mathbb{R}$, its asymptotic expansion at $\infty$ is $\sum_{k=0}^{\infty} b_{k} x^{-k}$ if

$$
\lim _{x \rightarrow \infty} x^{n}\left(f(x)-\sum_{k=0}^{n} \frac{b_{k}}{x^{k}}\right)=0 \quad \text { for all } n \in \mathbb{N} .
$$

The usual notation is $f(x) \sim \sum_{k=0}^{\infty} b_{k} x^{-k}$. Furthermore, the notation

$$
f(x) \sim g(x) \sum_{k=0}^{\infty} b_{k} x^{-k}
$$

means $f(x) / g(x) \sim \sum_{k=0}^{\infty} b_{k} x^{-k}$. In this case,

$$
\lim _{x \rightarrow \infty}\left(\frac{f(x)}{g(x)}-b_{0}\right)=0 .
$$

This fact is also denoted as $f(x) \sim b_{0} g(x)$ at $\infty$, and $b_{0} g(x)$ is said to be the dominant term or to represent the leading behavior of $f(x)$ at infinity.

Lemma 4.2. The modified Bessel function $I_{v}(x)$ of (4-6) satisfies

$$
I_{v}^{\prime}(x)=I_{\nu-1}(x)-\frac{v}{x} I_{\nu}(x), \quad I_{v}(x) \sim \frac{e^{x}}{\sqrt{2 \pi x}} \text { at } \infty .
$$

Lemma 4.3. Let $h_{a}$ be given by expression (4-4) with $g_{1}$ given in (4-7).

(i) The function $h_{a}$ is an even function.

(ii) In a neighborhood of zero,

$$
h_{a}(x)=-\frac{2^{13 / 8}}{3^{9 / 8}} \frac{a^{9 / 4}}{\Gamma\left(\frac{1}{4}\right)}+m(a) x^{2}+O\left(x^{4}\right),
$$

where $m(a)$ is positive for $a>0$.

(iii) For $a>\sqrt[6]{27 / 2}$, we have $\lim _{x \rightarrow+\infty} h_{a}(x)=-\infty$.

(iv) For $a<\sqrt[6]{27 / 2}$, we have $\lim _{x \rightarrow+\infty} h_{a}(x)=+\infty$.

(v) If the system of equations $h_{a}(x)=h_{a}^{\prime}(x)=0$ has some positive solution, it has to be at the point

$$
x=x^{*}(a):=\frac{3 a}{\sqrt{2 a^{6}-27}} .
$$

(vi) For $x>0$ and sufficiently large values of $a$, the function $h_{a}(x)$ does not vanish. Proof. (i) From its definition, it is clear that $h_{a}$ is even. Hence, from now on we will consider only positive values of $x$. 
(ii) By Lemma 4.2, it is easy to verify that

$$
h_{a}(x)=\frac{x^{3 / 2}}{9}\left(9 I_{1 / 4}\left(\frac{a x^{2}}{\sqrt{6}}\right)-\sqrt{6} a^{3} I_{-3 / 4}\left(\frac{a x^{2}}{\sqrt{6}}\right)\right) .
$$

By using this last expression and (4-6), the Taylor expansion of $h_{a}$ at the origin follows.

(iii), (iv) By applying the second part of Lemma 4.2 to the last expression of $h_{a}$ and by the asymptotic expansion properties, we obtain

$$
h_{a}(x) \sim \frac{1}{\sqrt{2 \pi}} \frac{\left(18-2 \sqrt{6} a^{3}\right) x^{2} e^{a x^{2} / \sqrt{6}}}{18 x^{3 / 2}} \text { at } \infty .
$$

Hence the results about the behavior of $h_{a}$ at infinity are proved.

(v) Let $\bar{x}(a)$ be a solution of the system of equations $h_{a}(x)=h_{a}^{\prime}(x)=0$. Since $h_{a}$ satisfies the linear ordinary differential equation (4-5), we have when $x=\bar{x}(a)$, then $\left(\left(6 a^{6}-81\right) x^{2}-27 a^{2}\right) h_{a}^{\prime \prime}(x)$ has to be zero. Assume that at this point $h_{a}^{\prime \prime}$ also vanishes, and that $\bar{x}(a) \neq x^{*}(a)$. Then, since $\bar{x}(a)$ is not a singular point for (4-5), this implies that $h_{a}(x) \equiv 0$, which gives a contradiction. Therefore $\bar{x}(a)$ has to be the positive root of $\left(\left(6 a^{6}-81\right) x^{2}-27 a^{2}\right)=0$, say $x^{*}(a)$, as we wanted to prove.

(vi) Let $\tilde{x}(a)$ be a positive solution of $h_{a}(x)=0$. Then $x=\tilde{x}(a)$ is also a solution of the equation

$$
\frac{I_{1 / 4}\left(\frac{a x^{2}}{\sqrt{6}}\right)}{I_{-3 / 4}\left(\frac{a x^{2}}{\sqrt{6}}\right)}=\frac{\sqrt{6} a^{3}}{9} .
$$

If for sufficiently large $a$ such a solution $\tilde{x}(a)$ exists, there are two possibilities:

(a) either $a \tilde{x}(a)^{2}$ is bounded above when $a$ tends to infinity,

(b) or $a \tilde{x}(a)^{2}$ is unbounded.

In the first case we can construct a sequence $\left\{a_{n}\right\}_{n}$ tending to infinity and such that $\lim _{n \rightarrow \infty} a_{n} \tilde{x}\left(a_{n}\right)^{2}=k \geq 0$. By replacing these values in (4-9) we arrive at a contradiction.

In the case (b), by Lemma $4.2, I_{1 / 4}$ and $I_{-3 / 4}$ have the same behavior at infinity. Therefore, the left hand side of Equation (4-9) tends to 1 when $a_{n} \tilde{x}\left(a_{n}\right)^{2}$ tends to infinity. However, the right hand side tends to infinity, again reaching a contradiction. Hence, for sufficiently large $a$, the function $h_{a}$ does not vanish, as we wanted to prove.

The main result of this subsection is this: 
Proposition 4.4. Let $\varphi$ be the function

$$
\varphi(a):=3 \sqrt{3} I_{1 / 4}\left(\frac{3 \sqrt{3 / 2} a^{3}}{2 a^{6}-27}\right)-\sqrt{2} a^{3} I_{-3 / 4}\left(\frac{3 \sqrt{3 / 2} a^{3}}{2 a^{6}-27}\right) .
$$

Denote by $\bar{a}$ the largest positive solution of the equation $\varphi(a)=0$. Then the differential equation (4-2) has no limit cycles for $a \geq \bar{a}$.

Proof. To prove that system (4-2) has no limit cycles for some value of $a$, it suffices to show that its corresponding $h_{a}$ does not change sign. For sufficiently large $a$, by Lemma 4.3(vi), we already know that this is true. On the other hand, by studying the behavior of $h_{a}$ near zero and infinity (see again Lemma 4.3), it is clear that $h_{a}$ changes sign for $a<\sqrt[6]{27 / 2}$. Hence the case $a \geq \sqrt[6]{27 / 2}$ remains to be studied. Let $\tilde{a}$ be the biggest value of $a$ for which the function $h_{a}$ has some zero. Denote any of these zeros by $z(\tilde{a})$. From the behavior of $h_{a}$ near zero and infinity, and from the regularity of $h_{a}$ with respect to $x$ and $a$, we have $h_{\tilde{a}}(z(\tilde{a}))=h_{\tilde{a}}^{\prime}(z(\tilde{a}))=0$. Hence, by Lemma 4.3(v),

$$
z(\tilde{a})=x^{*}(\tilde{a}):=\frac{3 \tilde{a}}{\sqrt{2 \tilde{a}^{6}-27}} .
$$

By imposing that $h_{\tilde{a}}\left(x^{*}(\tilde{a})\right)=0$ in the expression for $h_{a}$ given in (4-8), we get the desired expression for $\varphi$.

Remark 4.5. Although we have not been able to perform the analytic study of the zeros of $\varphi$, it is not difficult to make a numerical study. The equation $\varphi(a)=0$ has a unique positive solution $\bar{a} \simeq 1.636$. For this value of $a$, the corresponding value of $d=-a^{2}$ in (4-1) is $\bar{d} \simeq-2.678$.

Uniqueness of limit cycles. From Lemma 4.1, we have to study (4-1) only in the case $d<0$. As in the van der Pol equation, for obtaining the uniqueness of the limit cycle with our procedure, it suffices to apply Theorem A with $n=2$. Following the notation introduced in Theorem A we get

$$
M_{2}(x)=3 d g_{2}^{\prime \prime}(x)+9 x g_{2}^{\prime}(x)+4 d^{2} x^{2} g_{2}(x),
$$

where $g_{2}(x)$ is any solution of the linear ordinary differential equation

$$
y^{\prime \prime \prime}(x)+2 d x^{2} y^{\prime}(x)+\left(\frac{8}{3} d-4 x^{2}\right) x y(x)=0 .
$$

Hence, to prove the existence of at most one limit cycle and its hyperbolicity, it suffices to choose a concrete $g_{2}$ as the solution of (4-12), such that its associated $M_{2}$, given in (4-11), does not change sign.

All the solutions of (4-12) are analytic for all $x \in \mathbb{R}$, with an infinite radius of convergence. We will choose the even solution of (4-12) defined by the initial 
conditions

$$
y(0)=1, \quad y^{\prime}(0)=y^{\prime \prime}(0)=0 .
$$

Notice that this function $g_{2}$ produces an even function $M_{2}$. Before studying the sign of $M_{2}$ we need to study the function $g_{2}$ near infinity in some detail. Recall that $g_{2}$ is a solution of Equation (4-12). It is easy to see that infinity is a singularity for Equation (4-12). Although unfortunately not regular, ${ }^{1}$ the singularity turns out to be of normal irregular type. For this kind of singularity there are some powerful results, which in most cases give the asymptotic expansions of a fundamental set of solutions. The result we need here is a generalization of a theorem of Poincaré; a proof can be found in [Horn 1901]. See also [Wasow 1965, Theorem 12.3].

Theorem 4.6. Consider the linear ordinary differential equation

$$
y^{(n)}(x)+b_{1}(x) y^{(n-1)}(x)+\cdots+b_{n}(x) y(x)=0,
$$

where the functions $b_{s}(x)$ are either rational functions or admit asymptotic expansions at infinity of the form

$$
b_{s}(x) \sim x^{s k} \sum_{i=0}^{\infty} \frac{b_{s, i}}{x^{i}}, \quad s=1,2, \ldots, n,
$$

where $k$ is a positive integer or zero. Assume that the algebraic equation

$$
m^{n}+b_{1,0} m^{n-1}+\cdots+b_{n-1,0} m+b_{n, 0}=0
$$

associated with (4-14) has $n$ different roots $m_{1}, m_{2}, \ldots, m_{n}$. Then (4-14) has $n$ linearly independent solutions $y_{1}, y_{2}, \ldots, y_{n}$ whose asymptotic expansions at infinity are of the form

$$
y_{s}(x) \sim e^{f_{s}(x)} x^{\alpha_{s}} \sum_{i=0}^{\infty} \frac{B_{s, i}}{x^{i}}, \quad s=1,2, \ldots, n,
$$

where $\alpha_{s}$ and $B_{s, i}$ are constants with $B_{s, 0}=1$, and the $f_{s}$ are polynomials in $x$ of degree $k+1$, vanishing at zero and having leading coefficient $m_{s} /(k+1)$. The asymptotic expansions of the functions $y_{s}(x)$ can be uniquely determined by formal substitutions in (4-14).

(A more general result about irregular singularities of linear equations, including also the case of multiple roots, can be found in [Wasow 1965, Theorem 19.1]. See [Bender and Orszag 1999, Chapter 3; Erdélyi 1956, Chapter III; Ince 1927,

\footnotetext{
${ }^{1}$ When infinity is a regular singularity for a linear equation, generically the solutions of the equation for sufficiently large $x$ are $x^{\alpha} \sum_{k=0}^{\infty} b_{k} x^{-k}$, where $\alpha$ is not necessarily an integer, and the series has a positive radius of convergence; in nongeneric cases, some logarithms can appear in the expressions of the solutions.
} 
Chapters VII and XVII] for more examples of irregular singularities, both normal and not; for a more recent point of view about linear differential equations, see [Varadarajan 1996] and the references therein.)

The next lemma gives the desired properties of $g_{2}$ at infinity.

Lemma 4.7. Consider $-\sqrt[3]{27 / 2}<d<0$. Let $g_{2}$ be the solution of (4-12) with initial conditions given in (4-13). Then the following conditions hold:

(i) The function $g_{2}$ is defined for all $x \in \mathbb{R}$, and it is an even positive function.

(ii) The functions $g_{2}^{\prime}(x)$ and $g_{2}^{\prime \prime}(x)$ are positive for all $x>0$.

(iii) The dominant term of the asymptotic behavior of $g_{2}$ at infinity is

$$
g_{2}(x) \sim c_{1} e^{r_{1} x^{2}} x^{\alpha_{1}},
$$

where $r_{1}$ is the positive root of $2 r^{3}+d r-1=0, \alpha_{1}=-\frac{2}{3} \frac{2 d+9 r_{1}^{2}}{d+6 r_{1}^{2}}$, and $c_{1}$
is a positive constant.

Proof. (i) From the initial conditions that $g_{2}$ satisfies, we get

$$
g_{2}(x)=\sum_{n=0}^{\infty} a_{n}\left(x^{2}\right)^{n}
$$

where $a_{0}=1, a_{1}=0, a_{2}=-d / 9$, and

$$
a_{n}=\frac{1}{n(n-1)(2 n-1)}\left(a_{n-3}-\frac{1}{3} d(3 n-4) a_{n-2}\right), \quad \text { for } n \geq 3 .
$$

Furthermore, since $a_{n}>0$ for $n \geq 2$, item (i) holds. Item (ii) follows by taking derivatives of the expression of $g_{2}$.

We prove item (iii) in two steps: first we find a basis of formal solutions of (4-12), then we use Theorem 4.6 and formal computations to get the leading term of the asymptotic behavior of $g_{2}$.

We start our first step by using a heuristic method, called the method of dominant balance, to get the leading terms of the basis of formal solutions; see for instance [Bender and Orszag 1999, p. 76]. Apply to (4-12) the change of dependent variable $y(x)=e^{S(x)}$, which yields

$$
3 S^{\prime \prime \prime}(x)+9 S^{\prime}(x) S^{\prime \prime}(x)+3 S^{\prime}(x)^{3}+6 d x^{2} S^{\prime}(x)-12 x^{3}+8 d x=0 .
$$

The leading behavior of $g_{2}(x)$ will be determined by those contributions to $S(x)$ that do not tend to zero when $x$ approaches the irregular singularity. We suppose that the dominant terms in this equation when $x$ is sufficiently large are $3 S^{\prime}(x)^{3}, 6 d x^{2} S^{\prime}(x)$ and $-12 x^{3}$. Then we obtain the simplified equation $3 S^{\prime}(x)^{3}+$ $6 d x^{2} S^{\prime}(x)-12 x^{3}=0$, whose solutions are $S_{s}(x)=r_{s} x^{2}+p_{s}(s=1,2,3)$, where the $r_{s}$ are the three roots of the equation $2 r^{3}+d r-1=0$ and the $p_{s}$ 
are arbitrary constants that we take as zero for simplicity. Afterwards, we can verify that when we replace these expressions of $S_{s}(x)$ in (4-17), the terms of the equation that we have neglected are, at infinity, of smaller order than those we have kept in the simplified equation. This fact validates the first step of the procedure. Once a value of $s=1,2,3$ is fixed, to obtain the next contribution to the leading behavior we introduce the new change of variable $y(x)=e^{r_{s} x^{2}+S(x)}$, with $\lim _{x \rightarrow \infty} S(x) / x^{2}=0$. We apply the method of dominant balance again (for brevity we omit the full differential equation satisfied for this new $S$ ). We propose the simplified equation $3\left(2 d+12 r_{s}^{2}\right) x^{2} S^{\prime}(x)+\left(36 r_{s}^{2}+8 d\right) x=0$, whose solution is $S(x)=\alpha_{s} \log x$, with $\alpha_{s}=-\left(4 d+18 r_{s}^{2}\right) /\left(3 d+18 r_{s}^{2}\right)$, where again the additive constant is not taken into account. As before, we can verify the self-consistency of the calculations by replacing this expression of $S(x)$ in the complete equation. A third term is obtained by using the change of variable $y(x)=e^{r_{s} x^{2}+\alpha_{s} \log x+S(x)}$, with $\lim _{x \rightarrow \infty} S(x) / \log x=0$. Repeating the same procedure, we obtain a solution that does not contribute to the leading behavior. Hence our candidates to be the leading behaviors at infinity of a basis of formal solutions of (4-12) are $e^{r_{s} x^{2}} x^{\alpha_{s}}$, where $s=1,2,3$. To end this step we show that (4-12) admits three formal solutions of the form

$$
\hat{y}_{s}(x)=e^{r_{s} x^{2}} x^{\alpha_{s}} \sum_{i=0}^{\infty} \frac{C_{s, i}}{x^{2 i}},
$$

where the $C_{s, i}$, for $s=1,2,3$ and $i=1,2, \ldots$, are constant, with $C_{s, 0}=1$. To prove this last assertion, fix a value of $s$ and introduce in (4-12) the change of variables $y(x)=x^{\alpha_{s}} e^{r_{s} x^{2}} h(u)$, with $u=1 / x$. Then the function $h(u)$ must satisfy an equation with the structure

$b_{1} u^{9} h^{\prime \prime \prime}(u)+u^{6}\left(b_{2}+b_{3} u^{2}\right) h^{\prime \prime}(u)+u^{3}\left(b_{4}+b_{5} u^{2}+b_{6} u^{4}\right) h^{\prime}(u)+u^{4}\left(b_{7}+b_{8} u^{2}\right) h(u)=0$,

where the coefficients $b_{i}$ depend on the parameter $d$ and on $r_{s}$. It is straightforward to verify that this equation admits a formal solution of the form $h(u)=$ $\sum_{n=0}^{\infty} h_{n}\left(u^{2}\right)^{n}$ if and only if $b_{4}=54\left(\left(30 d^{3}-324\right) r_{s}^{2}-162 d^{2} r_{s}+108 d-d^{4}\right) \neq 0$. Since $r_{s}$ satisfies the equation $2 r^{3}+d r-1=0$ and $d \in(-\sqrt[3]{27 / 2}, 0)$, we have $b_{4} \neq 0$. Hence, for each $s,(4-12)$ admits the formal solution given in (4-18) as we wanted to prove. This is precisely the definition of a normal irregular singular point [Ince 1927, p. 168]. (The radius of convergence of these formal series is generally difficult to determine, and may be zero.)

For the second step in the proof of (iii), we check that (4-12) satisfies the assumptions of Theorem 4.6. Notice that

$$
b_{1}(x) \equiv 0, \quad b_{2}(x)=2 d x^{2}, \quad b_{3}(x)=x^{3}\left(-4+\frac{8 d}{3 x^{2}}\right) .
$$


Hence $k=1$, and the equation associated with (4-12) is $m^{3}+2 d m-4=0$. Therefore, the polynomials given in (4-15) are of the form $f_{s}(x)=m_{s} x^{2} / 2+n_{s} x$, where $m_{s}$, for $s=1,2,3$ are the three different roots of the previous cubic equation (notice that we need to use the interval of values where $d$ varies, and that in the left boundary of this interval, Theorem 4.6 is no longer applicable, because the cubic polynomial has a double root). If we write $f_{s}(x)=r_{s} x^{2}+n_{s} x$, then the values $r_{s}$ are in fact the roots of the cubic equation $2 r^{3}+d r-1=0$ obtained above by the dominant balance method. Notice also the approach above gives $n_{s}=0$, for $s=1,2,3$. Hence, by using Theorem 4.6 we can assure that Equation (4-12) has a basis of solutions $y_{s}$, for $s=1,2,3$, such that

$$
y_{s}(x) \sim \hat{y}_{s}(x)=e^{r_{s} x^{2}} x^{\alpha_{s}} \sum_{i=0}^{\infty} \frac{C_{s, i}}{x^{2 i}},
$$

with $C_{s, 0}=1$, and some constants $C_{s, i}$, where $s=1,2,3$ and $i=1,2, \ldots$

Now the function $g_{2}$ can be written as $g_{2}(x)=c_{1} y_{1}(x)+c_{2} y_{2}(x)+c_{3} y_{3}(x)$, for some constants $c_{s}(s=1,2,3)$. From (4-19), we have the leading behaviors at infinity of this basis of solutions. Since from items (i) and (ii) we know that the function $g_{2}(x)$ is a solution of (4-12) that tends to infinity when $x \rightarrow+\infty$, we conclude that the leading behavior of the asymptotic expansion of $g_{2}$ is $g_{2}(x) \sim$ $c_{1} e^{r_{1} x^{2}} x^{\alpha_{1}}$ for the value $c_{1}>0$ given above, where $r_{1}$ is the positive root of $2 r^{3}+$ $d r-1=0$ and $\alpha_{1}=-\left(4 d+18 r_{1}^{2}\right) /\left(3 d+18 r_{1}^{2}\right)$, as we wanted to prove.

The next lemma gives some properties for the function $M_{2}$. To stress its dependence with respect to $d$, we will rename it $M_{2, d}$. Hence

$$
M_{2, d}(x):=M_{2}(x)=3 d g_{2}^{\prime \prime}(x)+9 x g_{2}^{\prime}(x)+4 d^{2} x^{2} g_{2}(x) .
$$

Lemma 4.8. Consider $-\sqrt[3]{27 / 2}<d<0$. Let $M_{2, d}$ be given in (4-20), where $g_{2}$ is the solution of (4-12), with initial conditions given in (4-13). Then

(i) The function $M_{2, d}(x)$ is positive for $x \neq 0$ near 0 , and $M_{2, d}(0)=0$.

(ii) $\lim _{x \rightarrow+\infty} M_{2, d}(x)=+\infty$.

(iii) If $M_{2, d}^{\prime}\left(x_{1}\right)=0$ for some $x_{1}>0$, then $M_{2, d}\left(x_{1}\right)=\left(\left(\frac{2 d^{3}+27}{3}\right) x_{1}-\frac{3 d}{x_{1}}\right) g_{2}^{\prime}\left(x_{1}\right)>0$. Proof.

(i) Using the series expansion of $g_{2}(x)$ we obtain $M_{2, d}(x)=-d x^{4}+\left(\frac{9}{5}+\right.$ $\left.\frac{4}{27} d^{3}\right) x^{6}+0\left(x^{8}\right)$. Since $d$ is negative, we have $M_{2, d}(x)$ is positive for $x \neq 0$ near 0 , as we wanted to prove.

(ii) By using Lemma 4.7(iii), the leading term of the asymptotic expansion of $M_{2, d}$ at infinity is

$$
M_{2, d}(x) \sim c_{1} e^{r_{1} x^{2}} x^{2+\alpha_{1}}\left(4 d^{2}+18 r_{1}+12 d r_{1}^{2}\right),
$$


where $c_{1}, r_{1}$ and $\alpha_{1}$ are given also in that Lemma. Hence, because we have $4 d^{2}+$ $18 r_{1}+12 d r_{1}^{2}>0$, for the values of $d$ considered here, the result follows.

(iii) By taking the derivative of (4-20) with respect to $x$, we get an expression that also involves $g_{2}^{\prime \prime \prime}(x)$. Because $g_{2}(x)$ satisfies (4-12), we can simplify this expression. Finally, by evaluating the resulting expression at $x_{1}$, item (iii) follows since $x_{1}$ satisfies $M_{2, d}^{\prime}\left(x_{1}\right)=0$.

Using Lemmas 4.7 and 4.8, we get the main result of this section:

Proposition 4.9. System (4-1) has at most one limit cycle when $d \in(-\sqrt[3]{27 / 2}, 0)$. When it exists, the limit cycle is hyperbolic and unstable.

Proof. Recall that, for each value of $d$, by using Theorem A and the results of this subsection, we have reduced the problem to proving that the function $M_{2, d}(x)$ does not change sign. Recall also that $M_{2, d}$ is an even function vanishing at the origin, so it suffices to study it for positive values of $x$.

Consider, from now on, that $d$ is a fixed value in $(-\sqrt[3]{27 / 2}, 0)$. As straightforward consequences of Lemma 4.8, we have:

(a) For $x$ positive and small enough, $M_{2, d}$ is positive.

(b) For $x$ positive and large enough, $M_{2, d}$ is positive.

(c) If $M_{2, d}$ has a local minimum at some value $\bar{x}>0$, the function evaluated at this minimum $\bar{x}$ takes a positive value.

We claim that $M_{2, d}$ is positive for $x>0$. Assume for a contradiction that it takes a negative value for some $x^{*}>0$. By items (a) and (b), an absolute minimum $\bar{x}>0$ exists, at which of course $M_{2, d}(\bar{x})<0$. But this inequality contradicts (c). Hence $M_{2, d}$ is always positive or zero, as we wanted to prove.

\section{Conclusions}

Collecting the results obtained earlier, we conclude that system (4-1) has:

(i) No limit cycles when $d \geq 0$ or $d<-2.679$ (this value being obtained numerically by solving a nonlinear equation).

(ii) At most one limit cycle when $0>d>-\sqrt[3]{27 / 2} \simeq-2.381$.

We have not been able to cover all the values of the parameter $d$. There is a small gap for which we do not know the maximum number of limit cycles of system (4-1). By a numerical study, we conclude that the limit cycle is unique and exists only when $-2.198<d<0$. Hence, although we have not completely solved the problem, it seems the method presented in this paper gives reasonably good estimates for the regions of nonexistence of limit cycles, and for the regions of uniqueness of limit cycles. 


\section{References}

[Bender and Orszag 1999] C. M. Bender and S. A. Orszag, Advanced mathematical methods for scientists and engineers, I: Asymptotic methods and perturbation theory, Springer, New York, 1999. MR 2000m:34116 Zbl 0938.34001

[Cherkas 1997] L. A. Cherkas, "The Dulac function for polynomial autonomous systems on a plane”, Differ. Uravn. 33:5 (1997), 689-699. In Russian; translated in Differ. Equations 33 (1997), 692-701. MR 99a:34076 Zbl 0911.34026

[Cherkas and Grin' 1997] L. A. Cherkas and A. A. Grin', “A second-degree polynomial Dulac function for a cubic system on the plane", Differ. Uravn. 33:10 (1997), 1435-1436. In Russian; translated in Differ. Equations 33 (1997), 1443-1445. MR 99k:34055 Zbl 0929.34027

[Cherkas and Grin' 1998] L. A. Cherkas and A. A. Grin', "A Dulac function in a half-plane in the form of a polynomial of the second degree for a quadratic system", Differ. Uravn. 34:10 (1998), 1346-1348. In Russian; translated in Differ. Equations 34 (1998), 1346-1348. MR 2000g:34043 Zbl 0955.34017

[Cima et al. 1997] A. Cima, A. Gasull, and F. Mañosas, "Limit cycles for vector fields with homogeneous components”, Appl. Math. (Warsaw) 24:3 (1997), 281-287. MR 98c:34042 Zbl 0880.34032

[Duff 1953] G. F. D. Duff, "Limit-cycles and rotated vector fields", Ann. of Math. (2) 57 (1953), 15-31. MR 14,751c Zbl 0050.09103

[Erdélyi 1956] A. Erdélyi, Asymptotic expansions, Dover, New York, 1956. MR 17,1202c Zbl 0070. 29002

[Gasull and Giacomini 2002] A. Gasull and H. Giacomini, "A new criterion for controlling the number of limit cycles of some generalized Liénard equations", J. Differential Equations 185:1 (2002), 54-73. MR 2003i:34072 Zbl 1032.34028

[Horn 1901] J. Horn, "Uber die asymptotische Darstellung der Integrale linearer Differentialgleichungen”, Acta Math. 24 (1901), 289-308. Zbl 32.0326.01

[Ince 1927] E. L. Ince, Ordinary differential equations, Longmans, London, 1927. Reprinted by Dover, New York, 1944. MR 6,65f Zbl 0063.02971

[Lloyd 1979] N. G. Lloyd, "A note on the number of limit cycles in certain two-dimensional systems”, J. London Math. Soc. (2) 20:2 (1979), 277-286. MR 80k:34039 Zbl 0407.34027

[Perko 1975] L. M. Perko, "Rotated vector fields and the global behavior of limit cycles for a class of quadratic systems in the plane", J. Differential Equations 18 (1975), 63-86. MR 51 \#10752 Zbl 0297.34024

[Perko 1990a] L. M. Perko, "Global families of limit cycles of planar analytic systems", Trans. Amer. Math. Soc. 322:2 (1990), 627-656. MR 91c:58114 Zbl 0724.34033

[Perko 1990b] L. M. Perko, "Bifurcation of limit cycles", pp. 315-333 in Bifurcations of planar vector fields (Luminy, 1989), edited by J.-P. Françoise and R. Roussaire, Lecture Notes in Math. 1455, Springer, Berlin, 1990. MR 92c:58111 Zbl 0713.34037

[Varadarajan 1996] V. S. Varadarajan, "Linear meromorphic differential equations: a modern point of view”, Bull. Amer. Math. Soc. (N.S.) 33:1 (1996), 1-42. MR 96h:34011 Zbl 0862.34004

[Wasow 1965] W. Wasow, Asymptotic expansions for ordinary differential equations, Pure and Applied Mathematics 14, Wiley-Interscience, New York, 1965. MR 34 \#3041 Zbl 0133.35301

[Yamato 1979] K. Yamato, "An effective method of counting the number of limit cycles", Nagoya Math. J. 76 (1979), 35-114. MR 81a:58042 Zbl 0422.34036 
[Ye et al. 1986] Y. Q. Ye, S. L. Cai, L. S. Chen, K. C. Huang, D. J. Luo, Z. E. Ma, E. N. Wang, M. S. Wang, and X. A. Yang, Theory of limit cycles, Translations of Mathematical Monographs 66, American Mathematical Society, Providence, RI, 1986. MR 88e:58080 Zbl 0588.34022

[Zhang et al. 1992] Z. F. Zhang, T. R. Ding, W. Z. Huang, and Z. X. Dong, Qualitative theory of differential equations, Translations of Mathematical Monographs 101, American Mathematical Society, Providence, RI, 1992. MR 93h:34002 Zbl 0779.34001

Received November 20, 2004.

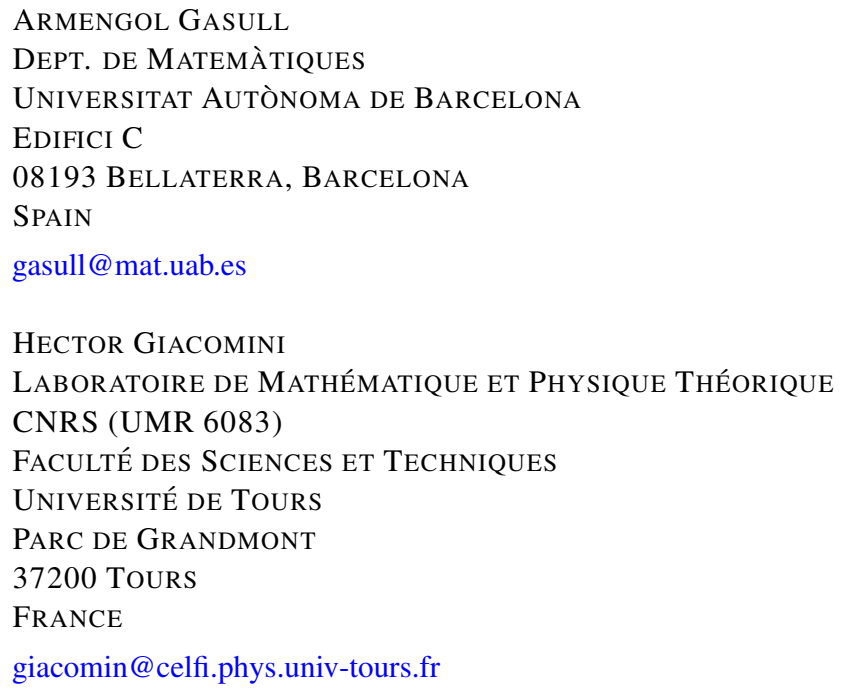

